\title{
Lubrication theory study with differentiated geometry parameterization and applications in hydrodynamic bearings
}

\author{
Júlia de Araújo Mota ${ }^{1}$ \\ PPGI/UFRJ, Rio de Janeiro, RJ. \\ Juliana Vianna Valério ${ }^{2}$ \\ IC/UFRJ, Rio de Janeiro, RJ.
}

\begin{abstract}
This article use the lubrication theory to simplify the Navier-Stokes equations for the flow between two cylinders with a small thickness of oil film. An effective parameterization of the geometry, proposed in Mota (2020) [6], is used to analyse traditional hydrodynamic bearings and two other configurations of them: elliptical and worn bearings. The simplifications in the model result in a elliptical partial differential equation, that are solved by the centered finite difference method which solution is the pressure field between the cylinders. The results agreed with the literature and different geometries are analysed. This modeling, as well as all its simulations were developed to integrate Ross-Rotordynamics, a library in Python for rotodynamic analysis, available on the GitHub platform.
\end{abstract}

Palavras-chave. Lubrication Theory, Geometry parameterization, Numerical Simulation, Hydrodynamic Bearings, Dynamic Coefficients.

\section{Introduction}

The Reynolds equations is widely used to understand the generation of pressure in lubricant theory. In this paper, dimensionless analysis will be performed in Navies-Stokes for the flow between two cylinders with a small thickness of lubricating oil film. An effective parameterization of the geometry is presented, enabling a more detailed description of the problem. The surfaces represent the stator and rotor of turbomachinery components such as bearings and seals. Instead of describing the geometry in terms of a gap function, this work describes the geometry of both the rotor and stator. Thus, different type of bearings can be modeled by simply defining the desired shape for each surface. Once the domain is defined, the pressure distribution of the fluid is obtained by means of the lubrication theory. First, the governing equations of a differential fluid element are determined by the Navier-Stokes equations and they are simplified to obtained the Reynolds equation, which solution is the pressure field of the fluid flow. Since this equation involves a discrete domain of the rotor and stator surfaces, it is solved by a finite differences method. The pressure field is then obtained and verified with the literature. This methodology is tested with three cases of study: a cylindrical bearing, a lemon bearing and a bearing with a worn stator.

The studies carried out by Pina and Carvalho (2006) [8], Andrade (2008) [1] and Queiroz (2018) [9] are relevant to this text because their work has inspired the parameterization used in the description of the geometry.

\footnotetext{
${ }^{1}$ juliadearaujomota@gmail.com.

2 juvianna@dcc.ufrj.br.
} 


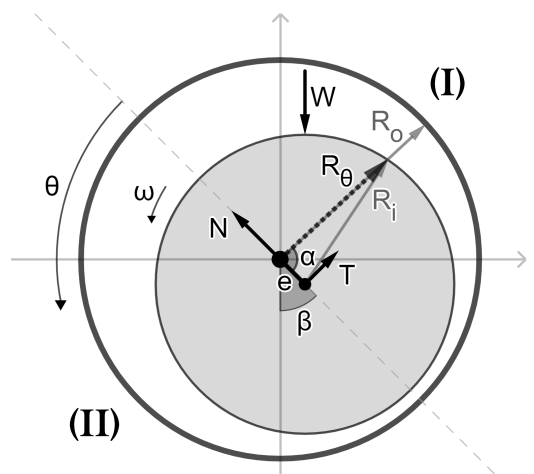

Figure 1: Schematic drawing of an eccentricity journal bearing

\section{Problem description}

To represent a hydrodynamic bearing, the dynamics of the fluid flow in the annular space between two cylinders is studied. The system is shown in Figure 1. The external cylinder, with radius $R_{o}$ is called stator and the internal cylinder, with radius $R_{i}$, is the rotor which spins with a speed $\omega$.

The rotation set forces that move the rotor to an eccentric position. According to Figure 1, any point in the rotor's surface can be described as $R_{\theta}=\sqrt{R_{i}^{2}-e^{2} \sin ^{2} \alpha}+e \cos \alpha$, with

$$
\alpha=\left\{\begin{array}{ll}
\frac{3 \pi}{2}-\theta+\beta, & \text { if } \frac{\pi}{2}+\beta \leq \theta<\frac{3 \pi}{2}+\beta \quad \text { (I) } \\
-\left(\frac{3 \pi}{2}-\theta+\beta\right), & \text { if } \frac{3 \pi}{2}+\beta \leq \theta<\frac{5 \pi}{2}+\beta \quad \text { (II) }
\end{array},\right.
$$

where $e$ is the eccentricity, $\beta$ is the attitude angle, $R_{o}$ is the stator's radius and $\theta$ is the angle used to define $R_{\theta}$. Note that $\theta$ is measured from an axis passing through the points of maximum and minimum fluid width. Also, it is important to note that the difference between the radius of the stator and the rotor is very small compared to the radius of the stator.

\section{Theoretical Modeling}

The dynamic of the fluid between the rotor and stator is modeled using the Navier-Stokes equations and the continuity equation. For this problem, the equations are

$$
\begin{aligned}
\rho\left(\frac{\partial \mathbf{v}}{\partial t}+\mathbf{v} \cdot \nabla \mathbf{v}\right) & =-\nabla p+\mu \nabla^{2} \mathbf{v} \\
\frac{\partial \rho}{\partial t}+\nabla \cdot(\rho \mathbf{v}) & =0
\end{aligned}
$$

where $\rho$ is the specific mass of the fluid and $\mu$ is the viscosity; $\mathbf{v}$ is the velocity field vector in cylindrical coordinates with components $u$ (axial), $v$ (radial) and $w$ (tangential); and the pressure field $p$.

Following the simplifications of the lubrication theory, the number of terms were reduced considerably. For the equation along the radial direction, the radial velocity disappeared and the 
pressure gradient along this direction is zero. Thus, the radial speed $v$ can be considered insignificant compared to the other speeds. Detail can be found in Mota (2020) [6]. In this way, Eq. (1a) can be rewritten as:

$$
\begin{array}{r}
-\frac{\partial p}{\partial z}+\mu\left[\frac{1}{r} \frac{\partial}{\partial r}\left(r \frac{\partial u}{\partial r}\right)\right]=0 \\
-\frac{1}{r} \frac{\partial p}{\partial \theta}+\mu\left[\frac{\partial}{\partial r}\left(\frac{1}{r} \frac{\partial(r w)}{\partial r}\right)\right]=0 .
\end{array}
$$

After the simplifications, Eqs. (2a-b) can be integrated in order to find the velocities $u$ and $w$ and using the boundary conditions: $u\left(R_{o}\right)=0, u\left(R_{\theta}\right)=0, w\left(R_{o}\right)=0$ and $w\left(R_{\theta}\right)=\omega R_{i}=W$ (where $W$ is tangential speed at the rotor's surface) the description of the velocities $u$ and $w$ is obtained as a function of the pressure gradient.

In order to obtain the pressure field, the continuity equation (1b) is integrated in the annular region of interest. After that, the pressure can be approximate by finite center difference scheme in the equation $(3)$ :

$$
\begin{gathered}
\frac{\partial}{\partial \theta}\left(C_{1} \frac{\partial p}{\partial \theta}\right)+\frac{\partial}{\partial z}\left(C_{2} \frac{\partial p}{\partial z}\right)=\frac{\partial}{\partial \theta} C_{0}, \\
\text { where } C_{0}=-\omega R_{i} R_{\theta}\left[\ln \left(\frac{R_{o}}{R_{\theta}}\right)\left(1+\frac{R_{\theta}^{2}}{\left(R_{o}^{2}-R_{\theta}^{2}\right)}\right)-\frac{1}{2}\right], \\
C_{1}=\frac{1}{4 \mu}\left[R_{o}^{2} \ln R_{o}-R_{\theta}^{2} \ln R_{\theta}-\left(R_{o}^{2}-R_{\theta}^{2}\right)(1+K)\right]-\frac{R_{\theta}^{2}}{2 \mu}\left[\left(\ln R_{\theta}-\frac{1}{2}+K\right) \ln \left(\frac{R_{o}}{R_{\theta}}\right)\right], \\
C_{2}=\frac{-R_{\theta}^{2}}{8 \mu}\left\{\left[R_{o}^{2}-R_{\theta}^{2}-\frac{\left(R_{o}^{4}-R_{\theta}^{4}\right)}{2 R_{\theta}^{2}}\right]+\left(\frac{R_{o}^{2}-R_{\theta}^{2}}{R_{r}^{2} \ln \left(R_{o} / R_{\theta}\right)}\right)\left[R_{o}^{2} \ln \left(\frac{R_{o}}{R_{\theta}}\right)-\frac{\left(R_{o}^{2}-R_{\theta}^{2}\right)}{2}\right]\right\} .
\end{gathered}
$$

\section{Results and Discussion}

The choice of parameterization of the model presented in this work allows easy adaptation to different geometries and operational conditions of rotating machine components. Once the numerical solution of the model has been obtained after the simplifications carried out throughout this text, it is possible to apply it to more specific contexts, allowing a greater exploration of the results.

The algorithm used was developed in Python, being one of the results of this research, and is available on the GitHub platform, through the software Ross-Rotordynamics [10], in which the authors of this text are co-authored. Currently in this software, the present model and all its functionalities correspond to the part called Fluid Flow, which is responsible for modeling the fluid film in bearings and provides the rest of the program with information necessary for the study of the stability of rotating dynamic systems.

\subsection{Short journal bearing}

In the literature, it is common to use the Reynolds equation after a series of simplifications to find the pressure field in the bearings. However, as the equation found can only be solved 


\begin{tabular}{c|c|c} 
& \multicolumn{2}{|c}{ Value } \\
\hline Element & Short bearing & Comparison \\
\hline Rotor radius $\left(R_{i}\right)$ & $0.2[\mathrm{~m}]$ & - \\
Stator radius $\left(R_{i}\right)$ & - & $0.015[\mathrm{~m}]$ \\
Gap $(F)$ & $0.0001[\mathrm{~m}]$ & $9 \cdot 10^{-5}[\mathrm{~m}]$ \\
Load $(W)$ & $50[N]$ & $100[N]$ \\
Length $L$ & $0.5[\mathrm{~m}]$ & $0.02[\mathrm{~m}]$ \\
Viscosity $(\mu)$ & $0.015[$ Pa.s $]$ & $0.05449[$ Pa.s $]$ \\
Rotation $(\omega)$ & $10.472[\mathrm{rad} / \mathrm{s}]$ & $50 \leq \omega \leq 550[\mathrm{rad} / \mathrm{s}]$
\end{tabular}

Table 1: Parameters used for the simulations.

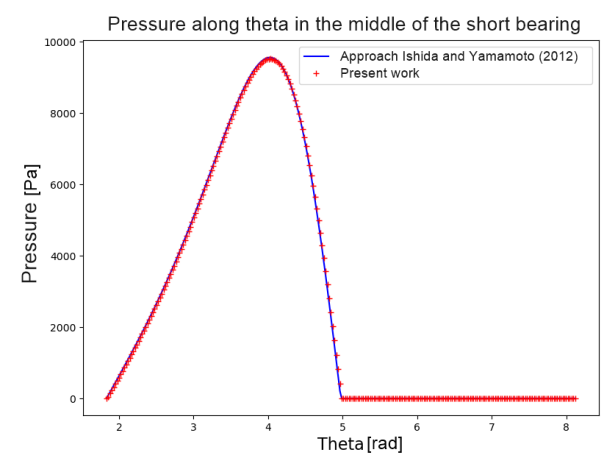

Figure 2: Pressure on a short journal bearing vs. angle $\theta$

numerically, they use the strategy of approximating the equation for bearings with short or infinitely long length. In this way, they find reduced models in which an analytical resolution is possible. In order to verify the simplifications that were made in this work, comparisons will be made with the results obtained by the current literature approximations.

According to Ishida and Yamamoto (2012) [4], to analyze the short journal bearing, the pressure variation in the $z$ direction is considered much larger than in the $\theta$ direction, i.e. $\partial p / \partial \theta \ll \partial p / \partial z$. Thus, the first term in the equation (3) is neglected and the approach formula for short journal bearing is obtained.

The numerical simulation of Table 1 generates a relative error $E \approx 10^{-3}$. The pressure behavior is reported with the result of the literature in Figure 2. This preliminary result is extremely relevant since this approach is used in several simulations for bearings, indicating that the model agrees with the literature.

\subsection{Comparison between geometries}

According to Ostayen and Beek (2009) [7], a component commonly found in high-speed machines is the elliptical bearing or "lemon bearing", as it is also known. It is a variation of the cylindrical bearing with axial groove and reduced clearance in one direction.

For the inclusion of this new geometry, adaptations to the stator radius are necessary, as it will no longer be constant in $\theta$. This new distance will be called $R_{o}^{*}=\sqrt{R_{o}^{2}-\epsilon^{2} \sin ^{2} \alpha}+\epsilon \cos \alpha$ and 


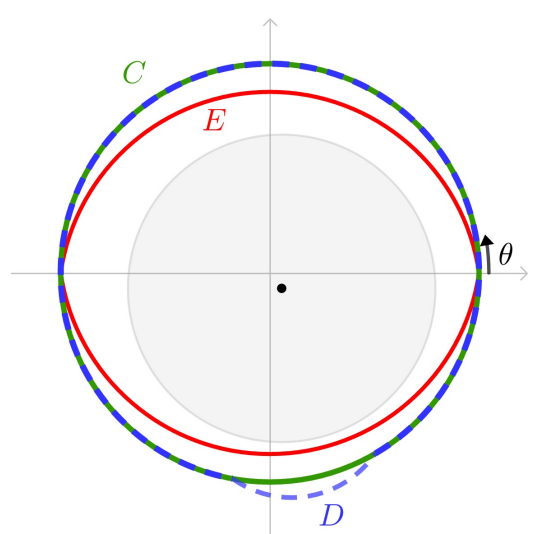

Figure 3: Schematic drawing listing the three bearing configurations shown.

varies along the angular position, where $\alpha=\left\{\begin{array}{ll}\pi / 2+\theta, & \text { se } \theta \in 1 \text { st quadrant } \\ 3 \pi / 2+\theta, & \text { se } \theta \in 2 \text { nd quadrant } \\ \theta-\pi / 2, & \text { se } \theta \in 3 \text { rd quadrant } \\ 5 \pi / 2-\theta, & \text { se } \theta \in 4 \text { th quadrant }\end{array}\right.$.

An important parameter to be defined is the $m$ preload which, in this text, will be established as: $m=\frac{\epsilon}{F}$ where $\epsilon$ is the ellipticity and $F=R_{o}-R_{i}$ is the radial clearance. For $m=0$, the bearing becomes cylindrical, while for $m \rightarrow 1$ the stator arcs tend to touch the axis.

Another important structure to be studied, is the bearing with wear. Although lubrication reduces friction between the metal surfaces of the bearing, these structures tend to wear out after a long period of operation or due to a number of repetitions of the starting cycles, where there is greater friction between the solid parts because there are still no forces of the fluid acting on the rotor. As the wear suffered by these components affects the radial clearance, they certainly influence the results of the pressure field and, consequently, the reaction forces and dynamic coefficients. For this reason, it is relevant to investigate the effect of wear, under certain operating conditions, on the dynamic response of the system.

The wear geometry that will be used in this text was adapted from the version presented by Machado and Cavalca (2015) [5]. The description was initially proposed by Dufrane, Kannel and McCloskey (1983) [2] and validated experimentally by Hashimoto, Wada and Nojima (2008) [3].

Considering that the fault starts at the angular position $\theta=\theta_{s}$ and ends at $\theta=\theta_{f}$, the stator description from the origin is defined as $R_{o}^{*}=R_{o}+d_{\theta}$, where

$d_{\theta}=\left\{\begin{array}{ll}0, & \text { se } 0 \leq \theta \leq \theta_{s}, \quad \theta_{f} \leq \theta \leq 2 \pi \\ d_{0}-F(1+\cos (\theta-\pi / 2)), & \text { se } \theta_{s}<\theta<\theta_{f}\end{array}\right.$.

In $\theta_{s}$ and $\theta_{f}$, the wear depth is zero, so the location of the edges can be defined as follows:

$$
\theta_{s}=\pi / 2+\cos ^{-1}\left(d_{0} / F-1\right)+\gamma \text { and } \theta_{f}=\pi / 2-\cos ^{-1}\left(d_{0} / F-1\right)+\gamma
$$

Large parts of today's rotating machines operate at high speeds of rotation. This speed influences the entire dynamic response of the bearing, from the equilibrium position to the stiffness and damping coefficients. For this reason, it is essential to understand how the model's responses behave for variations in rotation in each of the geometries presented.

In order to compare the results, the same simulation was used, which data are in Table 1, adapting only the specifics of each geometry and varying the speed of rotation in the $50 \leq \omega \leq$ $550[\mathrm{rad} / \mathrm{s}]$. For the elliptical bearing the preload $m=0.4$ was considered and, for the bearing with 

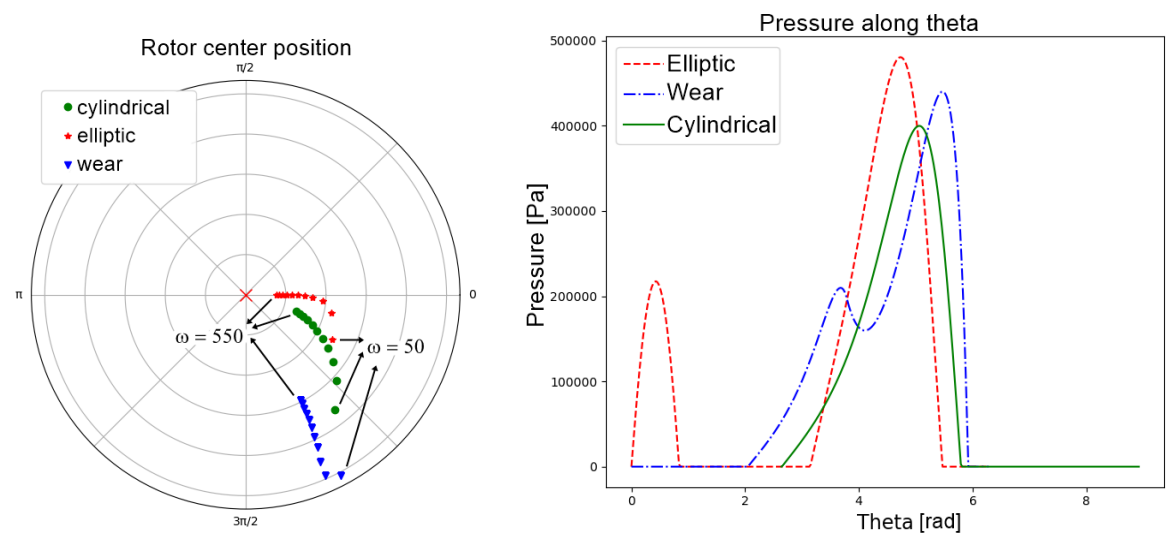

Figure 4: Results for different geometry configurations. The figure on the left shows the position of the center of the rotor by varying the speed. The figure on the right shows the pressures vs. angle $\theta$ in the middle of the bearing for the different configurations

wear, the maximum depth $d_{0}=50[\mu \mathrm{m}]$ with an angular displacement $\gamma=10^{\circ}$. The schematic drawing that relates the three bearing configurations is illustrated in Figure 3, in which the geometries are indicated by the letters $C, E$ and $D$, representing cylindrical, elliptical and worn bearings, respectively.

The first image in Figure 4 illustrates the variation of the equilibrium position for the cylindrical, elliptical and highlighted bearing. In the three cases, the eccentricity decreases with the increase of the rotation, however it is possible to observe that these approaches occur in different paths. While the cylindrical bearing moves in a semicircle-like path, the elliptical bearing generates a steeper curve, in which most points are aligned with the horizontal axis. On the other hand, the worn bearing has much higher eccentricities for all speeds. It is interesting to highlight the difference between the bearing balance positions with and without wear. Despite having very close geometry, wear alters the position of the rotor center and the way it behaves at different speeds.

As expected, the pressure behavior is changed for each geometry. The second image in Figure 4 demonstrates these changes to facilitate visualization. It is noticed that, in addition to the new curves, the maximum pressure value is also changed. The cylindrical bearing has the lowest pressure value, while the elliptical reaches the greatest magnitude, even though it is the configuration with the least eccentricity. It is also possible to notice that the wear, in addition to creating a new curve, causes the pressure results to be higher than in the cylindrical bearing, also modifying the place where the pressure reaches its highest value.

\section{Conclusions}

Considering the flow of a small thickness of lubricating oil film between two cylinders, the present work approximate pressure field and for that, the authors have used an effective parameterization of geometry, which enables a more detailed description of the problem, allowing to adapt the modeling of this work to other contexts. Without changing the proposed model, simulations were carried out in different configurations of hydrodynamic bearings. This parameterization also allows the model to be adapted in the future for many other geometries, including changes not only in the tangential direction, but also in the axial direction. In addition, following the base literature (Pina and Carvalho [8] and Andrade [1]), the equations were worked in cylindrical coordinates in 
order to preserve the effects of curvature, which may be interesting for future work . In this way, it is even feasible to make adaptations of the model to other components of rotating machines. The approximate pressure field was verified for cylindrical hydrodynamic bearings, comparing the responses of this model with those obtained through classic approaches in the literature. Two other configurations of hydrodynamic bearings were also presented: the elliptical and wear bearing. These new structures, obtained through changes only in the geometric description of the problem, without changing the modeling, portray the gain resulting from the chosen parameterization. In each situation, the impact of these changes on the dynamic response of the system was analyzed and, finally, comparisons were made between the three geometries, exploring the changes in the responses for different rotation speeds.

\section{Acknowledgements}

The code used for the simulations presented in this paper is part of Ross-Rotordynamics. We thank the team, especially David Maldonado and Thiago Ritto, for their important contributions.

\section{References}

[1] Andrade, S. F. A. Modelo Assintótico para Escoamento Monofásico em Bombas de Cavidades Progressivas. Dissertação de Mestrado - Departamentode Engenharia Mecânica, Pontifícia Universidade Católica do Rio de Janeiro, Riode Janeiro, 2008.

[2] Dufrane, K. F.; Kannel, J. W.; McCloskey, T. H. Wear of Steam Turbine Journal Bearings at Low Operating Speeds.J. of Lubrication Tech, [S.l.], v. 105,p. 313-317, 1983.

[3] Hashimoto, H.; WADA, S.; Nojima, K. Performance Characteristics of Worn Journal Bearings in Both Laminar and Turbulent Regimes. Part I: steady-state characteristics. ASLE Transactions, [S.1.], v. 29, p. 565-571, 2008.

[4] Ishida, Y.; Yamamoto, T. Flow-Induced Vibrations. In: Linear and Nonlinear Rotordynamics. Weinheim: Wiley Online Library, 2012. p. 235-261.

[5] Machado, T. H.; Cavalca, K. L. Modeling of Hydrodynamic Bearing Wearin Rotor-Bearing Systems. Mechanics Research Communications - Elsevier,[S.l.], v. 69, p. 15-23, 2015.

[6] Mota, J. A., Estudo da teoria de lubrificação com parametrização diferenciada da geometria e aplicações em mancais hidrodinâmicos. Dissertação (Mestrado em Informática) - PPGI, Universi-dade Federal do Rio de Janeiro, Rio de Janeiro, 2020.

[7] Ostayen, R. A.; Beek, A. Thermal Modelling of the Lemon-Bore Hydrodynamic Bearing.Tribology International - Elsevier, [S.l.], v. 42, p. 23-32, 2009.

[8] Pina, E. P.; Carvalho, M. S. Three-Dimensional Flow of a Newtonian Liquid Through an Annular Space with Axially Varying Eccentricity. Journal of Fluids Engineering, [S.l.], v. 128, n. 2, p. 223-231, 2006.

[9] Queiroz, L. H. M.Análise de Escoamento de Fluido no Interior de Componentes de Máquinas Rotativas. Projeto de Graduação - Curso de Engenharia Mecânica da Escola Politécnica, Universidade Federal do Rio de Janeiro, Rio de Janeiro, 2018.

[10] Timbó, R. et al. ROSS - Rotordynamic Open Source Software. Journal of Open Source Software, [S.l.], v. 5, n. 48, p. 2120, 2020. 